\title{
THE CHROMATIC SUM OF A GRAPH: HISTORY AND RECENT DEVELOPMENTS
}

\author{
EWA KUBICKA
}

Received 22 June 2003

\begin{abstract}
The chromatic sum of a graph is the smallest sum of colors among all proper colorings with natural numbers. The strength of a graph is the minimum number of colors necessary to obtain its chromatic sum. A natural generalization of chromatic sum is optimum cost chromatic partition (OCCP) problem, where the costs of colors can be arbitrary positive numbers. Existing results about chromatic sum, strength of a graph, and OCCP problem are presented together with some recent developments. The focus is on polynomial algorithms for some families of graphs and NP-completeness issues.
\end{abstract}

2000 Mathematics Subject Classification: 05C12, 05C05, 68Q25.

1. Introduction. Let $G=(V, E)$ be a graph with a vertex set $V$ and an edge set $E$. A proper coloring of the vertices of the graph $G$ is assigning different colors to adjacent vertices. The chromatic number $\chi(G)$ is the smallest number of colors in a proper coloring of $G$.

We start with two definitions which are central for this paper.

Definition 1.1. The chromatic sum of a graph $G, \sum(G)$, is the smallest sum of colors among all proper colorings with natural numbers.

DEFINITION 1.2. The strength of a graph $G, s(G)$, is the minimum number of colors necessary to obtain its chromatic sum.

To illustrate, consider the tree $T$ depicted in Figure 1.1. Notice that if only two colors are used, colors 1 and 2, then the sum of colors is 12 . However, by introducing a third color, color 3, the sum of colors drops down to 11 .

In fact, $\sum(T)=11, s(T)=3$, and $T$ is the smallest tree that requires three colors to achieve its chromatic sum.

2. Results for general graphs. It is well known that the chromatic number of a graph is an NP-complete problem. Finding the chromatic sum of a graph certainly has a similar flavor to finding the chromatic number. Therefore, the following result is not very surprising.

THEOREM 2.1 [11]. Chromatic sum problem is NP-complete.

Figure 1.1 depicts a tree $T$ with, obviously, chromatic number two and strength of three. As it turns out, the strength of a graph can exceed its chromatic number by an arbitrary large value. 


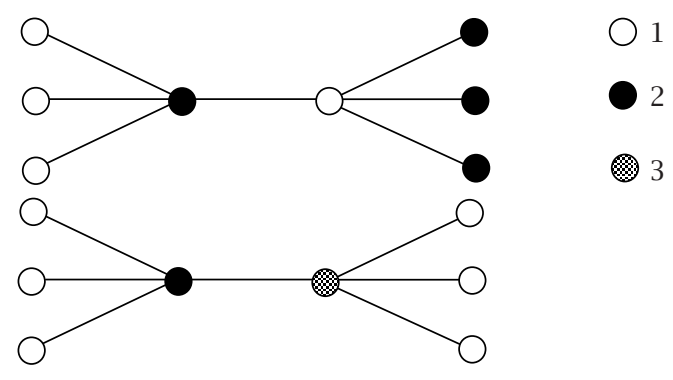

FiguRE 1.1. Tree $T$.

THEOREM 2.2 [1]. For every integer $k \geq 2$ and every positive integer $t$, there exists a graph $G_{k}^{t}$, with chromatic number $k$ and strength at least $k+t$.

The smallest order of a $G_{k}^{t}$ graph, that is, the smallest order of a $k$-chromatic graph which needs at least $k+t$ colors to achieve its chromatic sum, is denoted by $p(k, t)$. In [1], we have a rather complicated upper bound for $p(k, t)$ for any chromatic number $k$ and an easier upper bound for bipartite graphs.

THEOREM 2.3 [1].

$$
\begin{gathered}
p(k, t) \leq[k(k-1)+1]^{\lfloor t /(k-1)-1\rfloor}\left(k t_{\bmod (k-1)}+1\right) k^{3}, \\
p(2, t) \leq 8 \cdot 3^{t-1} .
\end{gathered}
$$

The above upper bound is not sharp. In fact, Hajiabolhassan et al. [3] have been able to improve the bound considerably by introducing a class of graphs called tabular graphs.

THEOREM 2.4 [3].

$$
p(k, t) \leq \frac{k(k+3)}{2}\left(\frac{k+1}{k-1}\right)^{t-1}-\left(\begin{array}{l}
k \\
2
\end{array}\right) .
$$

In the same paper, [3], the authors were able to give the exact value of $p(k, t)$ for $t$ fixed and $k$ sufficiently large.

THEOREM 2.5 [3]. For every integer $t \geq 1$, there exists a number $K$ such that for $k \geq K$,

$$
p(k, t)=k+t+t\left\lfloor\frac{1+\sqrt{8 k+1}}{2}\right\rfloor .
$$

In [18], Thomassen et al. showed several bounds for chromatic sum for general graphs. The first is a rather natural result of an application of a greedy algorithm: $\sum(G) \leq n+e$, where $n$ is the number of vertices in $G$ and $e$ is the number of edges. Here, we put vertices of $G$ into some order and, for each vertex $i$, consider its back degree $l_{i}$, 
that is, the number of edges to all the vertices listed before it. Notice that we can use one of $\left(l_{i}+1\right)$ colors to properly color vertex $i$. Therefore, $\sum(G) \leq \sum\left(l_{i}+1\right)=e+n$.

The second bound presented in [18] is not straightforward at all and gives an upper and lower limit for the chromatic sum in terms of $e$, the number of edges in $G$.

THEOREM 2.6 [18]. For any connected graph with e edges,

$$
\lceil\sqrt{8 e}\rceil \leq \sum(G) \leq\left\lfloor\frac{3}{2}(e+1)\right\rfloor
$$

both bounds are sharp.

We finish this section by giving a couple of general bounds for the strength of a graph, that is, the smallest number of colors needed to obtain the chromatic sum. The first one is a Brooks-type theorem and it involves the maximal degree of $G, \Delta(G)$.

THEOREM 2.7 [12].

$$
s(G) \leq \Delta(G)+1 .
$$

Moreover, $s(G)=\Delta(G)+1$ if and only if $G$ is a complete graph or an odd cycle.

Another interesting upper bound for the strength of a graph also involves $\Delta(G)$ together with another graph parameter called the coloring number, $\operatorname{col}(G)$. The coloring number of a graph $G, \operatorname{col}(G)$, also called the Szekeres-Wilf number, is the smallest number $d$ such that, for some linear ordering of vertices, "back" degree of every vertex is strictly smaller than $d$.

THEOREM 2.8 [4]. For any graph $G$,

$$
s(G) \leq\left\lceil\frac{\Delta(G)+\operatorname{col}(G)}{2}\right\rceil .
$$

Obviously, for every graph $G, \chi(G) \leq \operatorname{col}(G)$. However, the inequality $s(G) \leq \operatorname{col}(G)$ does not always hold. For example, for any tree $T, \operatorname{col}(T)=2$ and, as we will see later, most trees have strength larger than two. It can be shown though that aside from regular graphs, the inequality $\chi(G) \leq \operatorname{col}(G) \leq \Delta(G)$ is always true. This discussion, together with Theorem 2.8 , leads to the following conjecture.

CONJECTURE 2.9 [4]. For any graph $G$,

$$
s(G) \leq\left\lceil\frac{\Delta(G)+\chi(G)}{2}\right\rceil .
$$

3. Results for trees. Theorem 2.2 states that for every integer $k \geq 2$ and every positive integer $t$, there exists a graph $G_{k}^{t}$, with chromatic number $k$ and strength at least $k+t$. It has been chronologically preceded by a similar result just for trees.

THEOREM 3.1 [11]. For every natural positive integer $k$, there exists a tree with strength $k$. 


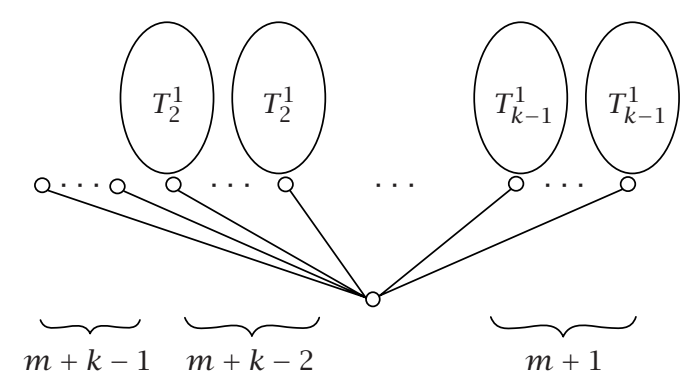

FIGURE 3.1. Tree $T_{k}^{m}$.

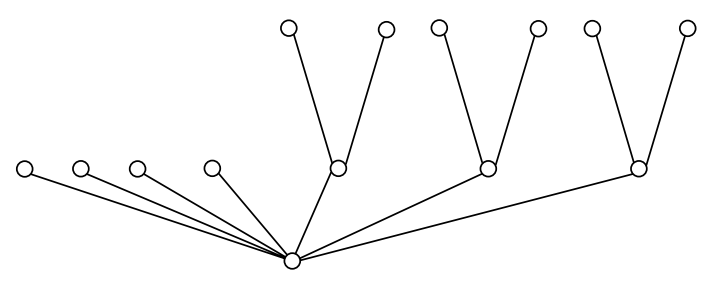

FiguRE 3.2. Tree $T_{3}^{2}$.

The order of the smallest such tree, $T_{k}$, is given by

$$
\left|T_{k}\right|=\frac{1}{\sqrt{2}}\left[(2+\sqrt{2})^{k-1}-(2-\sqrt{2})^{k-1}\right]
$$

The construction of the smallest tree of strength $k, T_{k}$, is based on the recursive construction of rooted trees $T_{k}^{m}$. The tree $T_{k}^{m}$ is defined to be the smallest rooted tree for which, in every coloring producing the chromatic sum, color $k$ is forced to appear at the root and any change of that color to a lower one must increase the sum of colors by at least $m$. The construction is depicted in Figure 3.1.

In Figure 3.2, we see the smallest rooted tree that needs color 3 to appear on the root. If either color 1 or 2 is used, the sum of colors increases by 2 .

Now, to obtain the tree $T_{k}$, we take two copies of the rooted trees $T_{k}^{2}$ and join their roots with an edge. In the above construction, the strength of trees $T_{k}, s\left(T_{k}\right)=k$ and the maximal degree $\Delta\left(T_{k}\right) \approx k^{2} / 2$. However, in Hajiabolhassan-Mehrabadi-Tusserkani bound, we have

$$
s(T) \leq\left\lceil\frac{\Delta(T)+\operatorname{col}(T)}{2}\right\rceil=1+\left\lceil\frac{\Delta(T)}{2}\right\rceil .
$$

Thus, for the trees $T_{k}$, this bound is not tight at all. Jiang and West [7] provided a different construction for trees of a given strength $k$, in which they minimized the maximal degree rather than order.

THEOREM 3.2 [7]. For every natural positive integer $k$, there exists a tree $S_{k}$ with $s\left(S_{k}\right)=k$ and $\Delta\left(S_{k}\right)=2 k-2$. 
Here, of course, we have

$$
s\left(S_{k}\right)=k \leq\left\lceil\frac{\Delta\left(S_{k}\right)+\operatorname{col}\left(S_{k}\right)}{2}\right\rceil=k,
$$

and the Hajiabolhassan-Mehrabadi-Tusserkani bound is sharp.

Another interesting bound for the strength of trees was given by Mitchem and Morriss.

THEOREM 3.3 [12]. Let $T$ be a tree and let $t$ be the number of vertices on the longest path in $T$. Then

$$
s(T) \leq 1+\left\lceil\frac{t}{2}\right\rceil
$$

If we fix the number of colors first and then find the minimum sum of colors over all proper colorings of $G$ using exactly these $k$ colors, then this sum is denoted by $\sum_{k}(G)$. We recall that the Grundy number is the largest number of colors in a proper coloring of $G$ in which every vertex of color $k>1$ is adjacent to at least one vertex of color $i$, for every $i<k$. The Grundy number is denoted by $\Gamma(G)$. We are now ready for our next definition.

Definition 3.4. The chromatic sequence for a graph $G$ is defined as follows:

$$
\sum_{X(G)}, \sum_{X(G)+1}, \sum_{X(G)+2}, \sum_{X(G)+3}, \ldots, \sum_{\Gamma(G)} .
$$

The chromatic sequence for trees is particularly interesting, as can be seen from the next theorem.

THEOREM 3.5 [10]. Chromatic sequence for trees is inverted unimodal, that is,

$$
\sum_{2}>\cdots>\sum_{s(T)-1}>\sum_{s(T)} \leq \sum_{s(T)+1}<\cdots<\sum_{\Gamma(T)}
$$

The above property is not true for general graphs. Moreover, there is no pattern for chromatic sequence for general graphs.

THEOREM 3.6 [10]. For any permutation $\pi$ of numbers 2 through $k$, there exists a graph $G$ which realizes this permutation in its chromatic sequence:

$$
\sum_{\pi(2)}(G) \leq \sum_{\pi(3)}(G) \leq \cdots \leq \sum_{\pi(k)}(G) .
$$

Moreover, the strict inequality or equality can be forced at each place in the above sequence.

4. Polynomial algorithms. There are not many known polynomial algorithms for computing chromatic sum for some families of graphs. Not surprisingly, there is an efficient algorithm for trees. There is also one for unicyclic graphs and one for outerplanar graphs. 
THEOREM 4.1 [11]. There is a linear algorithm to compute chromatic sum for trees.

The above algorithm proceeds from the leaves of a given tree to its root. It not only provides the chromatic sum for a given tree, but also gives the proper coloring of this tree, from which the chromatic sum results. A couple of generalizations of this algorithm was used in the next theorem. In one generalization, the algorithm produces several proper colorings of a tree with the smallest possible sum of colors and different colors used on the root of the tree (at least one of the sums is the chromatic sum). In the other generalization, the algorithm provides a proper coloring of a given tree with the smallest possible sum of colors and a given color forbidden on a given vertex of the tree. Before stating the next theorem, we recall two definitions. A unicyclic graph is a connected graph with exactly one cycle. An outerplanar graph is a graph having a planar imbedding with all its vertices lying on the boundary of the exterior region.

THEOREM 4.2 [9]. (i) There is a linear algorithm to compute chromatic sum for unicyclic graphs.

(ii) There is a cubic algorithm to compute chromatic sum for outerplanar graphs.

5. Interval graphs. We recall that an interval graph is an intersection graph of intervals on a real line. Unit (proper) interval graphs are defined to be intersection graphs of intervals, none of which contains another. Containment interval graphs are intersection graphs of intervals, any two of which either do not intersect or one contains another.

Nicoloso et al. [14] have a very interesting result concerning the strength of interval graphs.

THEOREM 5.1 [14]. Strength of a graph equals its chromatic number, $s(G)=\chi(G)$, for the following subfamilies of intersection graphs:

(i) unit interval graphs,

(ii) containment interval graphs,

(iii) intersection graphs of intervals of length not larger than 3.

Nicoloso et al. in the same paper, [14], constructed an infinite family of interval graphs $G^{[d]}$ with the property that $s\left(G^{[d]}\right) \geq 2 \chi\left(G^{[d]}\right)-1$.

The graph $G^{[d]}$ is an intersection graph of the set $B^{[d]}$ of intervals. The graph $G^{[d]}$ has the following other characteristics:

(i) order $n^{[d]}=6^{d-1}+2 \sum_{k=0}^{d-2} 6^{k}$;

(ii) the maximal degree, $\Delta\left(G^{[d]}\right)=n^{[d]} / 2$;

(iii) the chromatic number, $\chi\left(G^{[d]}\right)=\omega\left(G^{[d]}\right)=d$;

(iv) the Szekeres-Wilf number, $\operatorname{col}\left(G^{[d]}\right)=d-1$.

Later, Nicoloso [13] proved a result which gives a uniform bound for interval graphs and allows us to state precisely the strength of graphs $G^{[d]}$.

THEOREM 5.2 [13]. For an arbitrary interval graph $G$,

$$
s(G) \leq 2 \chi(G)-1 .
$$

So, for the family of interval graphs $\left\{G^{[d]}\right\}$, we actually have that $s\left(G^{[d]}\right)=2 \chi\left(G^{[d]}\right)-1$. 
As for an algorithm for computing the chromatic sum for general interval, the best known to date is an approximation algorithm due to Halldórsson et al. [5]. The algorithm is polynomial and has an approximation ratio of 1.665 .

6. Generalization of the chromatic sum and its applications. A very natural generalization of the chromatic sum would be to allow the costs of colors to be any numbers rather than consecutive natural numbers. In fact, that is the definition that appeared in a 1992 paper by Sen et al. [15].

DEFINITION 6.1 (optimum cost chromatic partition problem). Given a graph $G=$ $(V, E)$ with $n$ vertices and a sequence of coloring costs $\left(k_{1}, k_{2}, \ldots, k_{n}\right)$, find a feasible coloring $f(v)$ for each vertex $v$ such that the sum of coloring costs $\sum_{v \in V} k_{f(v)}$ is minimized.

There are many applications of the OCCP problem. The first one is due to Supowit in 1987 [16] and, of course, is not described using a graph theory language.

This is the following VLSI layout problem. A net consists of two groups of terminals (points on two opposite parallel lines) that need to be electrically connected. A routing segment is a line that connects two terminals of a net. Given a net list and an associated cost value for each layer, the objective is to partition the net list and place the partitions on different layers in such a way that routing segments do not cross on the same layer and that the total cost is minimum. This problem would correspond to the OCCP problem restricted to permutation graphs.

Another interesting application is given by Kroon et al. in their 1996 paper [8]. They show that OCCP problem for interval graphs is equivalent to the fixed interval scheduling problem with machine-dependent processing cost: each job $j$ must be executed during a given time interval $\left(s_{j}, f_{j}\right)$. We assume that a sufficient number of machines is available and each job must be executed by one of the machines. If job $j$ is executed by machine $m$, then the associated cost is $k_{m}$. The objective is to find a feasible schedule for all jobs with minimum total processing cost.

7. Perfect graphs. We recall that perfect graphs are those for which, for every induced subgraph $H$, the chromatic number is equal to the clique number, that is, $\chi(H)=$ $\omega(H)$. Perfect graphs form a widely studied family of graphs with many interesting subfamilies. Among the most studied subfamilies of perfect graphs are bipartite graphs, circle graphs, interval graphs, unimodular graphs, permutation graphs, and split graphs.

In 1984, Grötschel et al. [2] proved that finding chromatic number is solvable in polynomial time for perfect graphs. This result suggests that there may be some hope of constructing a polynomial algorithm for finding chromatic sum or solving the OCCP problems for a subfamily of perfect graphs. So far, unfortunately, the results were of negative nature.

First, we will look into the OCCP problem for subfamilies of perfect graphs.

THEOREM 7.1 [15]. OCCP problem for circle graphs is NP-complete.

Soon the result was followed by a similar one for interval graphs. 
THEOREM 7.2 [8]. OCCP problem for interval graphs is NP-complete.

In [6], Jansen proved several NP-completeness results for OCCP problem for many subfamilies of perfect graphs. Moreover, he showed that even approximating generalized chromatic sum is very hard.

THEOREM 7.3 [6]. OCCP problem for bipartite graphs is NP-complete. Moreover, for each $\varepsilon>0$, there exists no polynomial approximation algorithm with ratio $O\left(|V|^{0.5-\varepsilon}\right)$ for OCCP problem restricted to bipartite graphs unless $P=N P$.

This means that unless $P=N P$, there is no polynomial approximation algorithm $H$ which would compute a proper coloring with cost $H(I) \leq c \cdot O\left(|V|^{0.5-\varepsilon}\right) \cdot \mathrm{OPT}(I)$, where $c$ is a constant and $\operatorname{OPT}(I)$ is the minimum cost of a solution of instance $I$.

Next, in [6], Jansen gave a construction of a polynomial approximation algorithm with ratio $O\left(|V|^{0.5}\right)$ for OCCP problem restricted to bipartite graphs.

The result of Kroon et al. [8] for interval graphs was improved by Jansen by his corresponding result for approximation algorithm.

THEOREM 7.4 [6]. For each $\varepsilon>0$, there exists no polynomial approximation algorithm with ratio $O\left(|V|^{0.5-\varepsilon}\right)$ for OCCP problem restricted to interval graphs unless $P=N P$.

As in the case of bipartite graphs, Jansen [6] gives a construction of a polynomial approximation algorithm for solving OCCP problem for interval graphs with slightly relaxed ratio of $O\left(|V|^{0.5}\right)$. Similar results are obtained for permutation graphs and split graphs. A graph $G$ with vertices $v_{1}, \ldots, v_{n}$ and an edge set $E$ is a permutation graph if there exists a permutation $\pi$ with the following property: $(\pi(i)-\pi(j)) /(i-j)<0$ if and only if $\left(v_{i}, v_{j}\right) \in E$.

THEOREM 7.5 [6]. OCCP problem for permutation graphs or split graphs is NPcomplete.

Moreover, for each $\varepsilon>0$, there exists no polynomial approximation algorithm with ratio $O\left(|V|^{1-\varepsilon}\right)$ for OCCP problem restricted to permutation graphs or split graphs unless $P=N P$.

It is easy to see that the NP-completeness results for OCCP problem do not necessarily imply NP-completeness results for chromatic sum problem. In fact, in the proofs of the results in Theorems 7.2 through 7.5, the cost function plays a crucial role. For example, for bipartite graphs, Jansen [6] used the following cost functions: $c(1)=1, c(2)=10 \mathrm{~K}$, $c(3)=100 K^{2}, c(2)=1500 K^{3} n$, where $K$ is a constant and $n$ is the order of a graph.

Thus, a natural question occurs: for what subclasses of perfect graphs is the chromatic sum problem NP-complete? The first result is due to Szkaliczki [17].

THEOREM 7.6 [17]. The chromatic sum problem restricted to interval graphs is NPcomplete.

We will finish the survey by presenting a new result concerning split graphs. We recall that a graph is called a split graph if we can partition its vertex set into an independent set and a clique. 


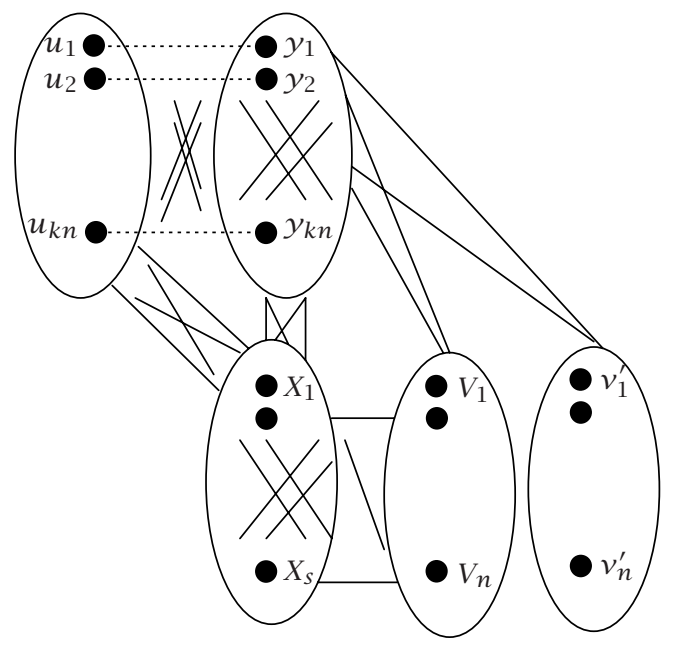

FiguRE 7.1. The sketch of the graph $G$.

THEOREM 7.7. The chromatic sum problem restricted to split graphs is NP-complete.

Proof. The proof will be done by reduction from minimum cover (MC) problem.

\section{MiNimum COVER (MC).}

GIVEN. A set $X=\{1,2, \ldots, n\}$, subsets $X_{i} \subset X$ for $1 \leq i \leq s$, and a positive integer $k$.

QUESTION. Do there exist $k$ subsets $X_{i_{1}}, X_{i_{2}}, \ldots, X_{i_{k}}$ with $\bigcup_{j=1}^{k} X_{i_{j}}=\{1,2, \ldots, n\}$ ? It is well known that MC problem is NP-complete.

Let $I$ be an instance of MC problem with the set $X=\{1,2, \ldots, n\}$, subsets $X_{i} \subset X$ for $1 \leq i \leq s$, and a constant $k$. We construct a split graph $G=(V, E)$ with $V=V_{1} \cup V_{2}$, where $V_{1}=\left\{v_{1}, \ldots, v_{n}\right\} \cup\left\{v_{1}^{\prime}, \ldots, v_{n}^{\prime}\right\} \cup\left\{u_{1}, \ldots, u_{k \cdot n}\right\}$ and $V_{2}=\left\{x_{1}, \ldots, x_{s}\right\} \cup\left\{y_{1}, \ldots, y_{k n}\right\}$. The vertices in $V_{1}$ form an independent set, while the vertices in $V_{2}$ form a clique. The neighborhood of every vertex $v_{i}^{\prime}$ is the same as the neighborhood of the corresponding vertex $v_{i}$. For every $i$ and $j$, the vertex $y_{j}$ is adjacent to $v_{i}$. The vertices $v_{1}, \ldots, v_{n}$ correspond to the set $X$ in the instance $I$ and $\left(x_{j}, v_{i}\right) \in E$ if and only if $i \notin X_{j}$. Every vertex $u_{j}, 1 \leq j \leq k n$, is adjacent to every vertex from $V_{2}$ except for the vertex $y_{j}$. The sketch of the graph $G$ is presented in Figure 7.1.

First notice that there exist $k$ subsets $X_{i_{1}}, X_{i_{2}}, \ldots, X_{i_{k}}$ with $\bigcup_{j=1}^{k} X_{i_{j}}=\{1,2, \ldots, n\}$ if and only if $G$ has a proper coloring that uses at most $k$ different colors on the set of vertices $\left\{v_{1}, \ldots, v_{n}\right\}$.

We define a constant $A$ in the following way:

$$
A=\frac{(k n+s)(k n+s+1)}{2}+\frac{n}{2}\left(n+2 k^{2}+3 k+2\right) .
$$

We will show that $G$ has a proper coloring that uses at most $k$ different colors on the set of vertices $\left\{v_{1}, \ldots, v_{n}\right\}$ if and only if the chromatic sum of $G$ is at most $A\left(\sum(G) \leq A\right)$. 
Assume that we can color $G$ properly with just $k$ colors on $\left\{v_{1}, \ldots, v_{n}\right\}$ (and therefore also on $\left.\left\{v_{1}^{\prime}, \ldots, v_{n}^{\prime}\right\}\right)$. We only consider colorings with minimum sum of colors. Then the sum of colors on $\left\{x_{1}, \ldots, x_{s}\right\} \cup\left\{y_{1}, \ldots, y_{k n}\right\}$ will be at most $2(((k+1) / 2) n)$. Since we need to use different colors on each vertex of the clique and, for every $i$, the vertex $u_{i}$ has to use the same color as $y_{i}$, we can bound the chromatic sum from above in the following way:

$$
\sum(G) \leq \frac{(k n+s)(k n+s+1)}{2}+2\left(\frac{k+1}{2} n\right)+(k+1)+\cdots+(k+k n)=A .
$$

Assume we need at least $k+1$ colors on $\left\{v_{1}, \ldots, v_{n}\right\}$. The cheapest way to color $G$ would then be to use as many ones as possible on $\left\{v_{1}, \ldots, v_{n}\right\}$ and use colors 2 through $(k+1)$ only once. Then, colors $(k+2)$ through $(k+k n)$ have to be used on vertices $\left\{u_{1}, \ldots, u_{k n}\right\}$ and all different colors on $\left\{x_{1}, \ldots, x_{s}\right\} \cup\left\{y_{1}, \ldots, y_{k n}\right\}$. Therefore, we have the following lower bound on the chromatic sum of $G$ :

$$
\begin{aligned}
\sum(G) \geq & \frac{(k n+s)(k n+s+1)}{2}+2[(n-k)+2+3+\cdots+k+(k+1)] \\
& +(k+2)+\cdots+(k+k n)=A+n+k^{2}+k>A .
\end{aligned}
$$

It still remains an open question whether the chromatic sum problem is NP-complete when restricted to bipartite graphs or permutation graphs.

\section{REFERENCES}

[1] P. Erdös, E. Kubicka, and A. J. Schwenk, Graphs that require many colors to achieve their chromatic sum, Congr. Numer. 71 (1990), 17-28.

[2] M. Grötschel, L. Lovász, and A. Schrijver, Polynomial algorithms for perfect graphs, Topics on Perfect Graphs, North-Holland Math. Stud., vol. 88, North-Holland, Amsterdam, 1984, pp. 325-356.

[3] H. Hajiabolhassan, M. L. Mehrabadi, and R. Tusserkani, Tabular graphs and chromatic sum, preprint, 1998.

[4] __ Minimal coloring and strength of graphs, Discrete Math. 215 (2000), no. 1-3, 265270.

[5] M. M. Halldórsson, G. Kortsarz, and H. Shachnai, Minimizing average completion of dedicated tasks and interval graphs, Approximation, Randomization, and Combinatorial Optimization (Berkeley, Calif, 2001), Lecture Notes in Computer Science, vol. 2129, Springer, Berlin, 2001, pp. 114-126.

[6] K. Jansen, Approximation results for the optimum cost chromatic partition problem, J. Algorithms 34 (2000), no. 1, 54-89.

[7] T. Jiang and D. B. West, Coloring of trees with minimum sum of colors, J. Graph Theory 32 (1999), no. 4, 354-358.

[8] L. G. Kroon, A. Sen, H. Deng, and A. Roy, The optimal cost chromatic partition problem for trees and interval graphs, Graph-Theoretic Concepts in Computer Science (Cadenabbia, 1996), Lecture Notes in Computer Science, vol. 1197, Springer, Berlin, 1997, pp. 279-292.

[9] E. Kubicka, Polynomial algorithm for finding chromatic sum for unicyclic and outerplanar graphs, to appear in Ars Combinatoria.

[10] _ Constraints on the chromatic sequence for trees and graphs, Congr. Numer. 76 (1990), 219-230.

[11] E. Kubicka and A. J. Schwenk, An introduction to chromatic sums, Proc. ACM Computer Science Conference (Louisville), ACM Press, New York, 1989, pp. 39-45. 
[12] J. Mitchem and P. Morriss, On the cost-chromatic number of graphs, Discrete Math. 171 (1997), no. 1-3, 201-211.

[13] S. Nicoloso, Sum coloring and interval graphs: a tight upper bound for the minimum number of colors, Discrete Math. 280 (2004), no. 1-3, 251-257.

[14] S. Nicoloso, M. Sarrafzadeh, and X. Song, On the sum coloring problem on interval graphs, Algorithmica 23 (1999), no. 2, 109-126.

[15] A. Sen, H. Deng, and S. Guha, On a graph partition problem with application to VLSI layout, Inform. Process. Lett. 43 (1992), no. 2, 87-94.

[16] K. Supowit, Finding a maximum planar subset of a set of nets in a channel, IEEE Trans. Computer-Aided Design 6 (1987), no. 1, 93-94.

[17] T. Szkaliczki, Routing with minimum wire length in the dogleg-free Manhattan model is NP-complete, SIAM J. Comput. 29 (1999), no. 1, 274-287.

[18] C. Thomassen, P. Erdös, Y. Alavi, P. J. Malde, and A. J. Schwenk, Tight bounds on the chromatic sum of a connected graph, J. Graph Theory 13 (1989), no. 3, 353-357.

Ewa Kubicka: Department of Mathematics, University of Louisville, Louisville, KY 40292, USA

E-mail address: ewa@1 oui svi11e.edu 


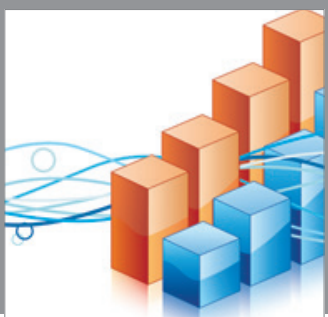

Advances in

Operations Research

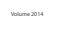

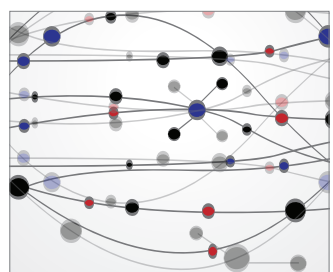

\section{The Scientific} World Journal
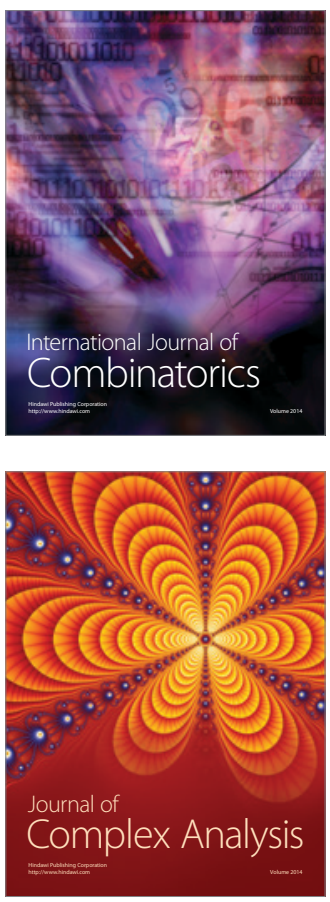

International Journal of

Mathematics and

Mathematical

Sciences
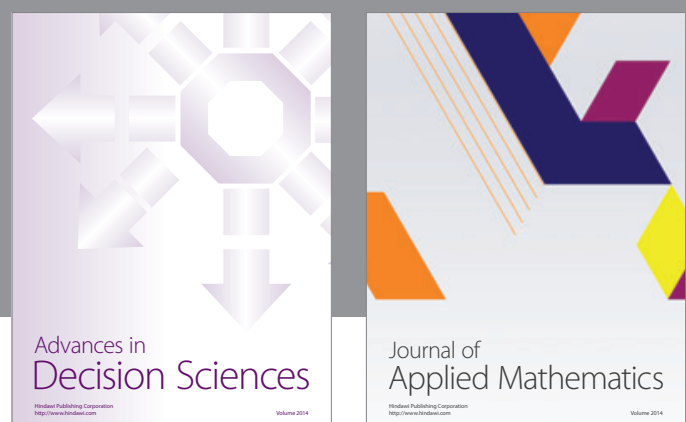

Journal of

Applied Mathematics
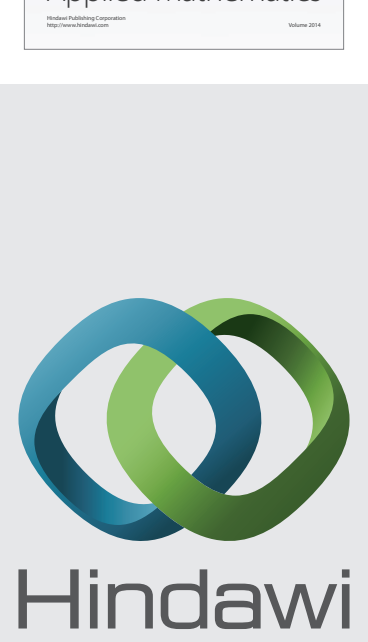

Submit your manuscripts at http://www.hindawi.com
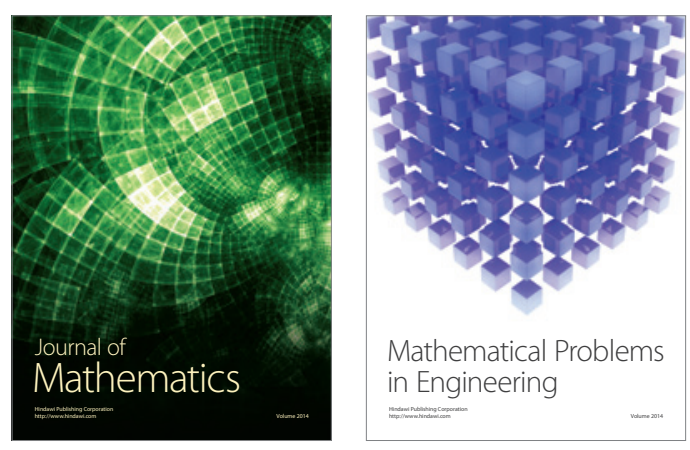

Mathematical Problems in Engineering
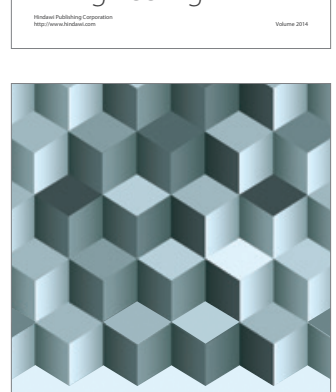

Journal of

Function Spaces
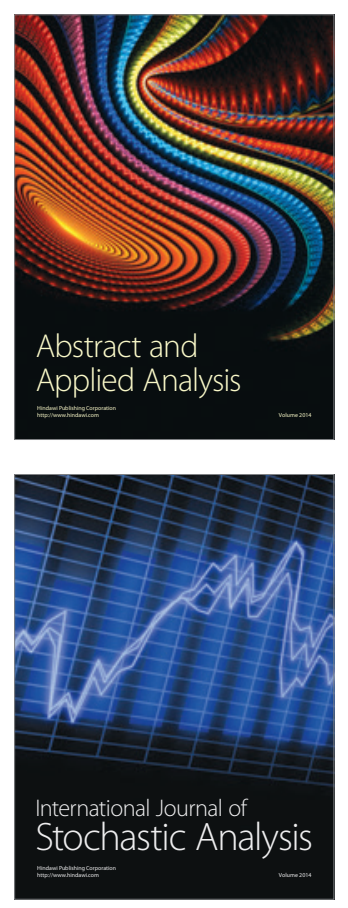

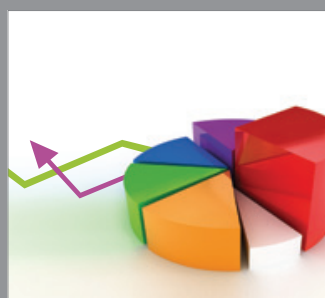

ournal of

Probability and Statistics

Promensencen
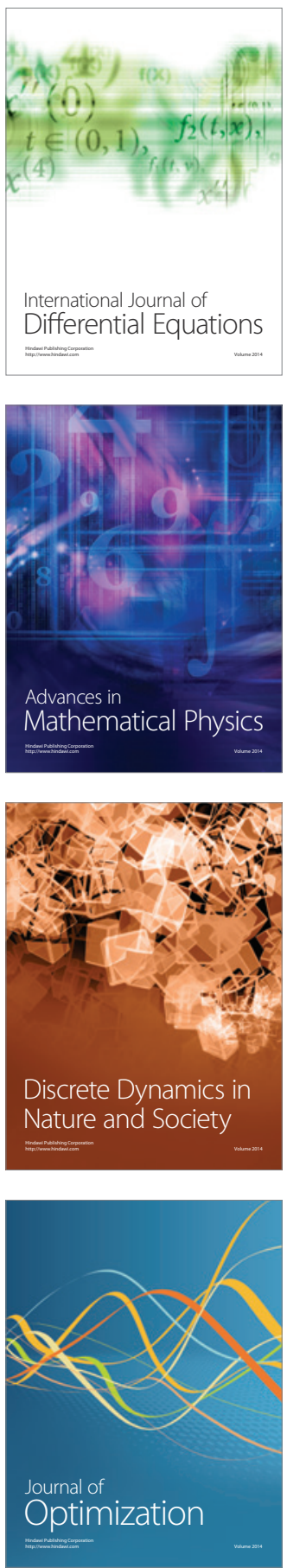